\title{
Differential microglia and macrophage profiles in human IDH- mutant and -wild type glioblastoma
}

\author{
Candice C. Poon ${ }^{1}$, Paul M.K. Gordon², Katherine Liu ${ }^{1}$, Runze Yang1, Susobhan \\ Sarkar ${ }^{1}$, Reza Mirzaei $^{1}$, Shiekh Tanveer Ahmad ${ }^{3}$, Martha L. Hughes ${ }^{1}$, V. Wee Yong ${ }^{1, *}$ \\ and John J.P. Kelly ${ }^{1, *}$ \\ ${ }^{1}$ Department of Clinical Neurosciences, University of Calgary, Calgary, AB, Canada \\ ${ }^{2}$ Centre for Health Genomics and Informatics, University of Calgary, Calgary, AB, Canada \\ ${ }^{3}$ Department of Pathology and Laboratory Medicine, University of Calgary, Calgary, AB, Canada \\ *These authors contributed equal supervision to this work \\ Correspondence to: John J.P. Kelly, email: jjkelly@ucalgary.ca \\ Keywords: glioblastoma; microglia; macrophages; isocitrate dehydrogenase; single-cell RNA sequencing \\ Received: January 11, $2019 \quad$ Accepted: March 04, $2019 \quad$ Published: May 03, 2019 \\ Copyright: Poon et al. This is an open-access article distributed under the terms of the Creative Commons Attribution License 3.0 \\ (CC BY 3.0), which permits unrestricted use, distribution, and reproduction in any medium, provided the original author and source \\ are credited.
}

\section{ABSTRACT}

Microglia and macrophages are the largest component of the inflammatory infiltrate in glioblastoma (GBM). However, whether there are differences in their representation and activity in the prognostically-favorable isocitrate dehydrogenase (IDH)-mutated compared to -wild type GBMs is unknown. Studies on human specimens of untreated IDH-mutant GBMs are rare given they comprise $10 \%$ of all GBMs and often present at lower grades, receiving treatments prior to dedifferentiation that can drastically alter microglia and macrophage phenotypes. We were able to obtain large samples of four previously untreated IDH-mutant GBM. Using flow cytometry, immunofluorescence techniques with automated segmentation protocols that quantify at the individual-cell level, and comparison between single-cell RNA-sequencing (scRNA-seq) databases of human GBM, we discerned dissimilarities between GBMassociated microglia and macrophages (GAMMs) in IDH-mutant and -wild type GBMs. We found there are significantly fewer GAMM in IDH-mutant GBMs, but they are more pro-inflammatory, suggesting this contributes to the better prognosis of these tumors. Our pro-inflammatory score which combines the expression of inflammatory markers (CD68/HLA-A, -B, -C/TNF/CD163/IL10/TGFB2), Iba1 intensity, and GAMM surface area also indicates that more pro-inflammatory GAMMs are associated with longer overall survival independent of IDH status. Interrogation of ScRNA-seq databases demonstrates microglia in IDH-mutants are mainly pro-inflammatory, while anti-inflammatory macrophages that upregulate genes such as FCER1G and TYROBP predominate in IDH-wild type GBM. Taken together, these observations are the first head-to-head comparison of GAMMs in treatment-naïve IDH-mutant versus -wild type GBMs. Our findings highlight biological disparities in the innate immune microenvironment related to IDH prognosis that can be exploited for therapeutic purposes. 


\section{INTRODUCTION}

Glioblastoma (GBM) is the most common adult brain cancer with a median survival of 14.6 months despite aggressive surgery and chemoradiation [1]. Success with immunotherapies such as checkpoint inhibitors in melanoma [2] and lung cancer [3] have prompted researchers to investigate its promise in GBM. However, none have proven efficacious. One possible reason why immunotherapies have failed to prolong survival in GBM is because they focus on modulating $\mathrm{T}$ cells, but $\mathrm{T}$ cells are sparse in GBM [4] unlike in melanoma [5] and lung cancer [6]. In general, the immune response in GBM is poorly understood and must be elucidated to develop effective treatments.

Single-cell RNA sequencing (scRNA-seq) studies of human GBM, in agreement with more classical immunohistochemical studies [7, 8], have shown that the predominant immune cell type in the tumor microenvironment are myeloid cells comprised of microglia and macrophages [4, 9-11]. It has been suggested that microglia and macrophages within GBM initially participate in tumor surveillance, but are subverted by GBM to adopt grossly anti-inflammatory phenotypes and subsequently promote immunosuppression, tumor angiogenesis and invasion [12]. Nevertheless, it is still unknown whether there is variation in the degree of immunosuppression experienced by GAMMs.

The majority of GAMM research has been in isocitrate dehydrogenase-wild type (IDH-WT) GBMs. In 2016, the World Health Organization Classification of Tumors of the Central Nervous System was revamped to divide GBM into three major categories: IDH-WT, IDHmutant (-MUT), and IDH not otherwise specified (when diagnostic procedures were lacking to determine IDH status)[13]. IDH-MUT GBMs have a better prognosis [14], are associated with different methylation patterns [15], and are present in younger patients compared to their wild type counterparts [16]. However, studies of untreated IDHMUT GAMMs are rare because not only do IDH-MUT GBMs account for approximately $10 \%$ of GBMs [16], but they almost always present first as lower grade gliomas which are treated with surgery and chemoradiation [17], processes which can drastically alter the native phenotype of microglia and macrophages [18, 19].

Our present study directly compares previously untreated human IDH-WT and -MUT GAMMs, representing a crucial step towards addressing the natural state of microglia and macrophages in these two potentially different microenvironments. We hypothesized that not only will there be heterogeneity in the microglia and macrophage response between GBMs, but that microglia and macrophages in IDHMUT tumors differ from those in wild type tumors. We found that innate immune cells are heterogeneously represented in wild type GBMs, while found in much smaller numbers in mutants. Notably, microglia and macrophages in mutants are of a more pro-inflammatory phenotype. Even wild type GBM patients with more pro-inflammatory microglia and macrophages had a longer overall survival. Furthermore, anti-inflammatory innate immune cells shared highly upregulated genes in common. These differences in innate immune biology can have important implications for development and selection of immunotherapy in GBM.

\section{RESULTS}

\section{Microglia and macrophage content is highly variable across IDH-wild type GBM and is decreased in IDH-mutants}

It is widely believed that microglia and macrophages make up one-third of all cells within GBM [20]. However, the origin of this estimate and applicability to all GBMs, particularly the newly diagnosed IDH-MUT GBMs, is unknown. Thus, we sought to determine GAMM content by using automated immunofluorescence segmentation techniques [21] with validation through flow cytometry. All GBM patients were previously untreated to allow characterization of the native microglia and macrophage state.

A surprisingly large range of CD11b $+\mathrm{CD} 45+$ cells was seen from approximately $0.0 \%$ to $65.4 \%$ of the parent population of IDH-WT GBM cells (Figure 2A). This substantial variation was again seen in matched GBMs (from $1.6 \pm 0.6 \%$ to $71.9 \pm 13.4 \%$ ) using Iba1 as a pan-microglia and macrophage marker (Figure $2 \mathrm{~B}$ and 2C). Different pan-microglia and macrophage markers (CD11b and CD45 double-positivity for flow cytometry, and Iba1 positivity for immunofluorescence) were used since dependence on only one microglia and macrophage marker for identification is insufficient. Furthermore, to ensure Ibal did not co-label with astrocytic markers such as glial fibrillary acidic protein (GFAP), a double immunofluorescent stain of Ibal and GFAP was performed in six GBMs (representative images are shown in Supplementary Figure 1). A positive correlation was observed between flow cytometry and immunofluorescence estimates of microglia and macrophage content (Pearson $\mathrm{r}=0.73, \mathrm{p}=0.03$; Figure 2D), thereby supporting the validity of our measurements.

A stark difference was observed between microglia and macrophage content in IDH-WT compared to MUT GBMs; mean frequency of IDH-WT GAMMs was $37.2 \pm 7.3 \%$ while it was $4.9 \pm 1.4 \%$ in IDH-MUTs $(p=0.02$; Figure $2 \mathrm{E}$ and $2 \mathrm{~F})$. Of note, as shown in the low magnification images in Figure 2B, microglia and macrophages were relatively evenly distributed across entire GBM sections instead of being in obvious clusters. 
Acquisition of patient samples

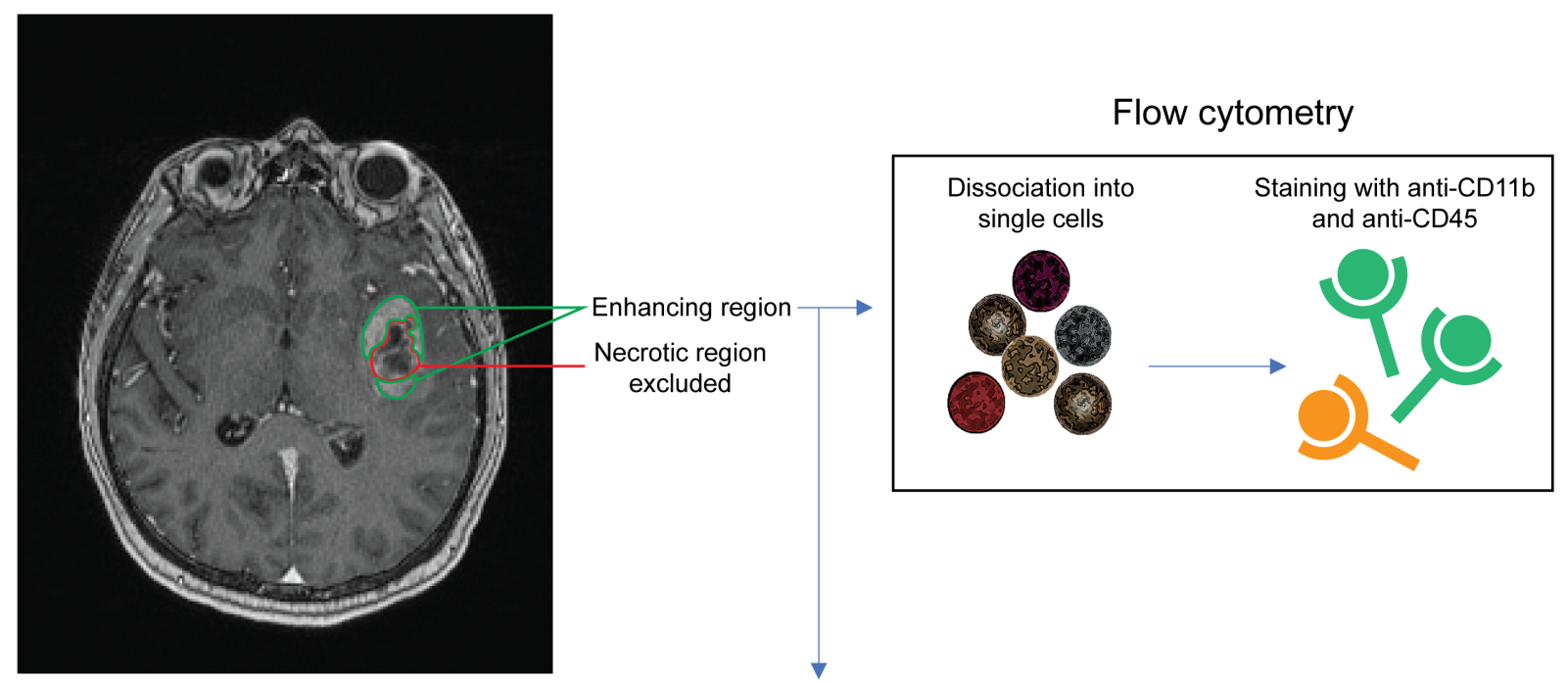

Automated quantitation of microglia/macrophage cell surface markers and secreted cytokines
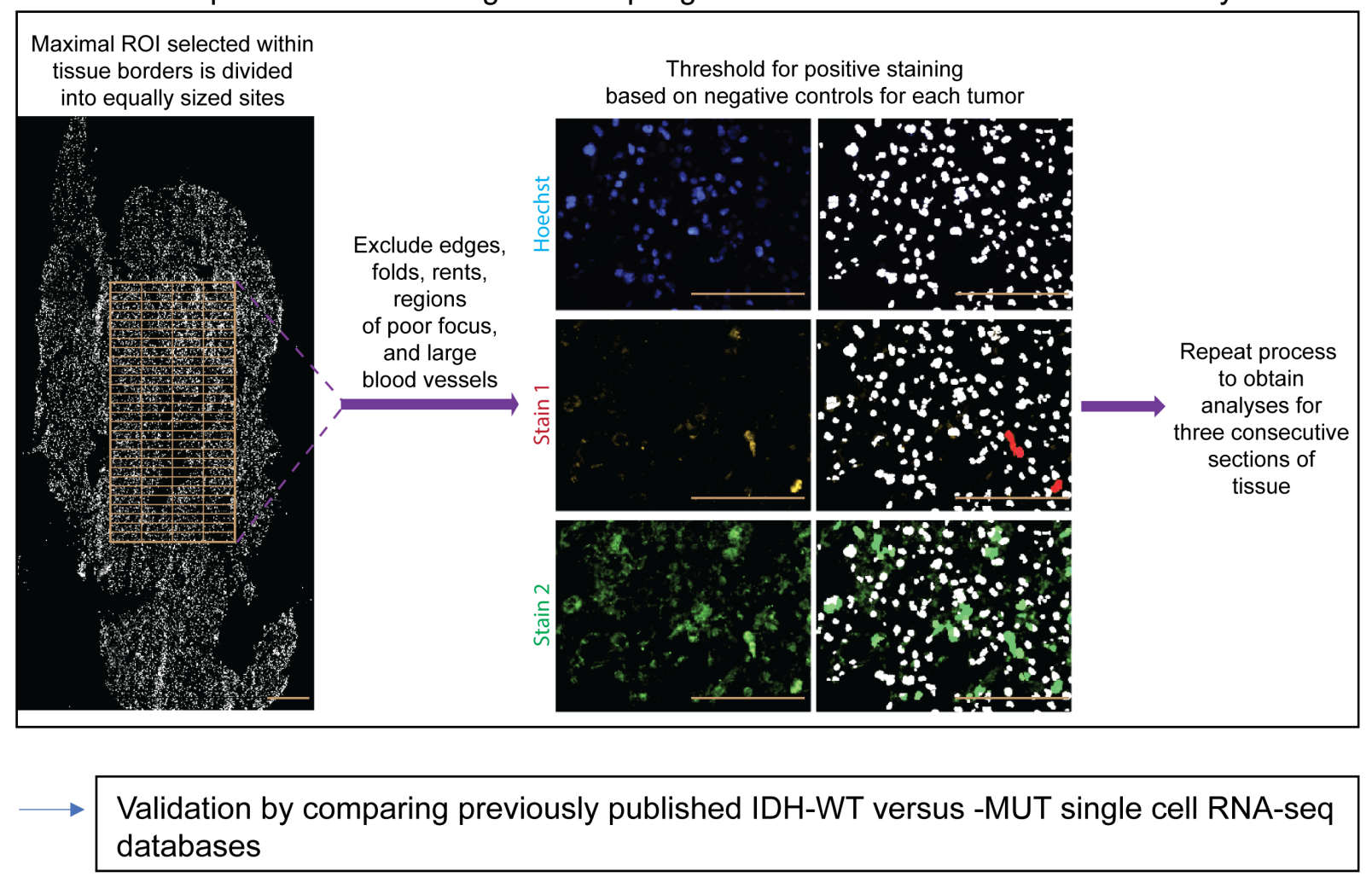

Figure 1: Schematic of methodology. Only untreated IDH-WT and -MUT GBMs were included in this study. A representative T1-weighted post-gadolinium MRI shows the enhancing region from which tissue was obtained. Areas of gross necrosis were avoided. Matched GBM samples were then analyzed via flow cytometry and immunofluorescence techniques. An automated image acquisition and segmentation protocol (see Methods) was used to quantitate microglia and macrophages. Finally, results were independently validated and further refined using bioinformatics comparison of scRNA-seq databases of IDH-MUTand -WT astrocytomas, with inclusion of only those tumors that were GBMs. Scale bars are $100 \mu \mathrm{m}$. 


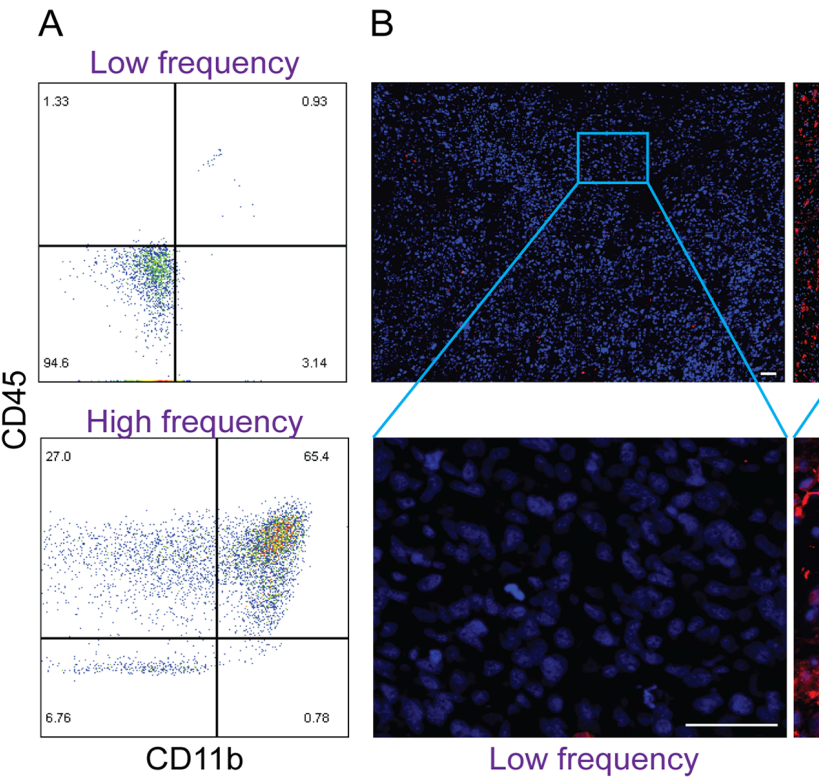

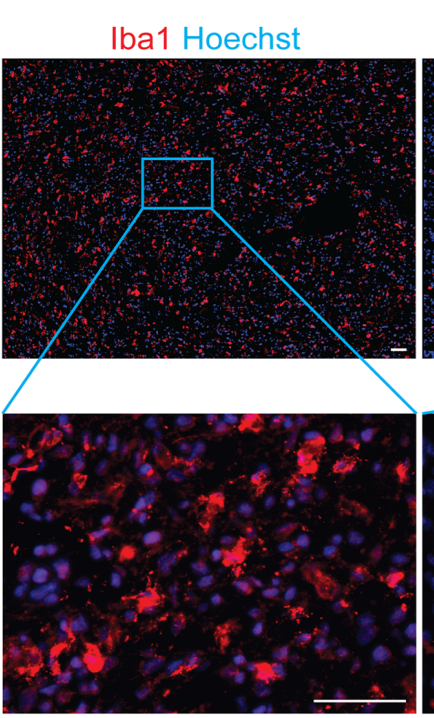

High frequency

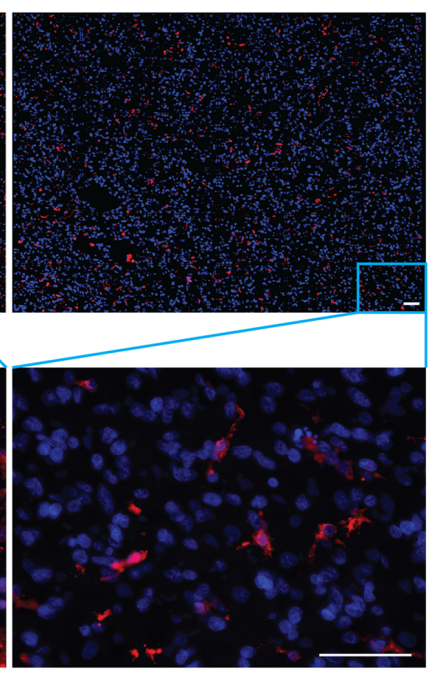

IDH-MUT GBM

D
E

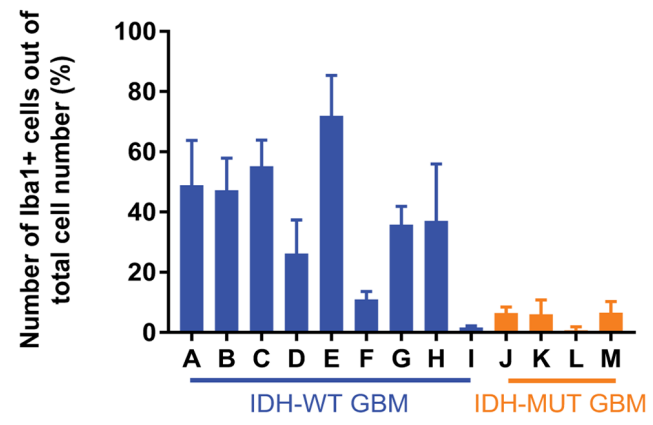

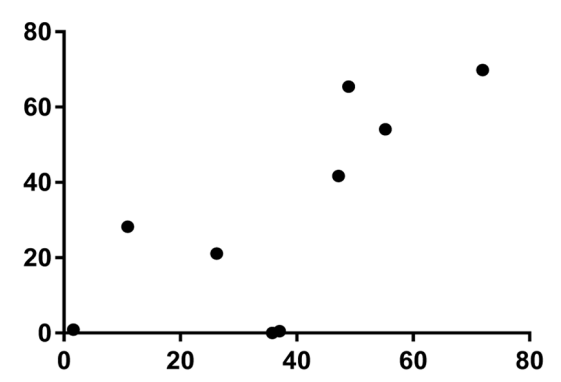

Iba1+ Cells according to immunofluorescence

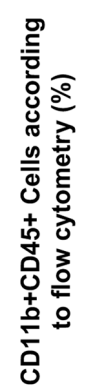

$\mathrm{F}$

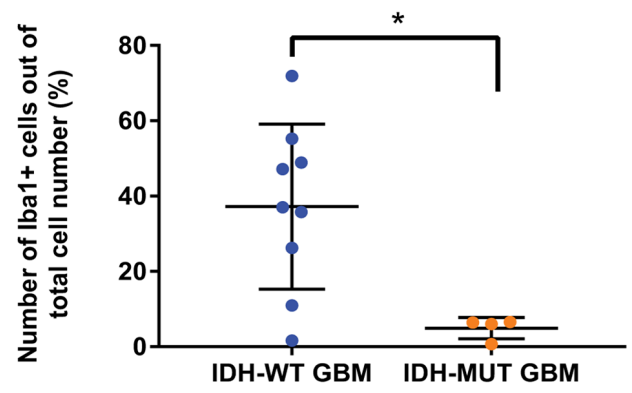

Figure 2: While the proportion of GAMM varies considerably across IDH-wild type GBMs, there is consistently less GAMM in IDH-mutant GBMs. (A) Flow cytometry reveals that microglia and macrophage (CD11b+CD45+; upper right quadrant) content in GBMs can range from almost none (top panel) to approximately 65\% of the tumor (bottom panel). (B) Immunofluorescence of tissue sections verifies the heterogeneity of microglia and macrophage (Iba1+) content in examples of low and high frequency in IDHwild type GBMs; an image from an IDH-mutant is also displayed. Scale bars are $50 \mu \mathrm{m}$. (C) Graphical representation and comparison of GAMM counts using both flow cytometry and immunofluorescence/automated segmentation techniques. (D) Correlation analysis reveals a significantly positive correlation between microglia and macrophage numbers obtained using flow cytometry and immunofluorescence (Pearson $r=0.73, p=0.03$ ). (E) Proportion of GAMM across GBMs, displaying heterogeneity in IDH-wild type tumors and consistently low numbers in IDH-mutants. Values for each tumor are mean $\pm \mathrm{SD}(\mathrm{n}=3$ sections for each GBM). (F) Evaluated as a group, there is a statistically significant difference in the amount of GAMM between IDH-wild type and -mutant GBMs $(\mathrm{p}=0.02)$. 


\section{Individual markers of GAMM activity are highly variable across specimens}

Next, we sought to investigate the inflammatory profile of GAMMs by interrogating three pro- and antiinflammatory markers each. While cell surface markers of inflammation are usually used, cytokines are one of the most salient indicators of inflammatory state. Thus, we incorporated a mixture of cell surface markers and cytokines in our inflammatory panels. In GBM, increased microglia and macrophage phagocytic ability, indicated by CD68, marks a more pro-inflammatory state [22]. Similarly, upregulation of HLA-A, -B, and -C results in more difficulty with immune evasion and is associated with a pro-inflammatory phenotype [23]. Lastly, we stained for tumor necrosis factor-alpha (TNF) because of its canonical pro-inflammatory nature and since it is the most widely used output of microglia and macrophage activation [24].

CD68 frequency in GAMMs ranged from $6.9 \pm 0.1 \%$ to $97.8 \pm 1.0 \%$ (Figure 3A-3D). A large range in expression was similarly seen with HLA-A, -B, -C $(19.3 \pm 6.6 \%$ to $95.8 \pm 5.2 \%$; Figure $3 \mathrm{E}-3 \mathrm{H})$ and $\mathrm{TNF}(5.4 \pm 0.2 \%$ to $63.2 \pm 7.9 \%$; Figure 3I-3L). While mean CD68 expression was approximately two-fold less frequent in IDH-MUT GAMMs (WT 51.2 $\pm 9.4 \%$ versus MUT 23.1 $\pm 8.2 \%$ ), and TNF expression two-fold higher (WT $19.7 \pm 6.1 \%$ versus MUT $39.4 \pm 6.6 \%$ ) than their wild type counterparts, these differences were not statistically significant $(p=0.09$ and $p=0.08$, respectively). The expression of HLA-A, $-\mathrm{B},-\mathrm{C}$ in microglia and macrophages was not statistically different between IDH-WT and IDH-MUT GAMMs (66.4 $\pm 6.9 \%$ versus $55.01 \pm 13.8 \%$, respectively, $\mathrm{p}=0.21$ ).

For our anti-inflammatory marker panel, we chose CD163 because it is often used as an anti-inflammatory marker in microglia and macrophages $[25,26]$. Interleukin-10 (IL10) and transforming growth factorbeta 2 (TGFB2) are major anti-inflammatory cytokines secreted by microglia and macrophages $[27,28]$ that are thought to heavily enforce the immunosuppressive microenvironment of GBM [29, 30]. Mean CD163+ GAMM frequency in IDH-MUT $(21.6 \pm 9.9 \%)$ was half that of IDH-WT $(48.4 \pm 8.9 \%)$ but this was not statistically significant $(\mathrm{p}=0.10 ;)$ (Figure 4A-4D). IL10 (WT $38.4 \pm 8.7 \%$ versus MUT $29.6 \pm 13.3 \%$ ) and TGFB2 (WT $35.4 \pm 9.7 \%$ versus MUT $44.5 \pm 11.3 \%, p=0.59)$ did not differ between the genotypes (Figure 4E-4L). Once again, incredible heterogeneity in the expression of these markers in GAMMs was seen both within IDH-WT GBMs and between IDH-WT and -MUT GBMs; CD163+ microglia and macrophages ranged from $3.4 \pm 5.1 \%$ to $100 \pm 0.0 \%$, IL10+ microglia and macrophages ranged from $1.4 \pm 4.5 \%$ to $82.3 \pm 6.5 \%$, and TGFB2+ microglia and macrophages ranged from $1.3 \pm 1.1 \%$ to $74.9 \pm 2.5 \%$.

In addition to inflammatory status, increased Iba1 intensity [31] and increased area [32] are also indicators of activation in microglia and macrophages. Hence, we used our automated segmentation protocol to quantify these parameters in GAMMs. IDH-MUT GAMMs reached an average of $112.7 \pm 23.5$ relative fluorescence units versus $60.0 \pm 19.4$ relative fluorescence units of IDHWT GAMMs, but this was not statistically significant $(p=0.14$; Figure 5A-5C). The mean area of IDH-MUT GAMMs was $53.3 \pm 8.9 \mu \mathrm{m}^{2}$ compared with $40.5 \pm 3.6 \mu \mathrm{m}^{2}$ of IDH-WT GAMMs ( $\mathrm{p}=0.13$; Figure 5B-5D).

In summary, the use of single markers to inform on the activity of GAMMs was not instructive, contributed by the large range of expression of each marker across specimens. Thus, we sought to combine these individual markers in a pooled analysis to inform on the overall activity of GAMMs.

\section{IDH-mutant glioblastoma-associated microglia and macrophages are more pro-inflammatory than those in IDH-wild type glioblastomas and pro-inflammatory status is associated with overall survival}

Many parameters have previously been associated with microglia and macrophage pro-inflammatory activation [33, 34]. While there is no one standard definition of activation, what is becoming clearer is that multiple parameters should be incorporated to determine the pro-inflammatory status of microglia and macrophages $[35,36]$. Thus, we chose to use a combination of cell surface markers, cytokines, Ibal intensity, and cell area. When all these characteristics are considered together, even though there are less GAMMs in IDH-MUT GBMs, they are more pro-inflammatory than in IDH-WT GBMs (124.5 \pm 21.6 pro-inflammatory units versus $54.0 \pm 15.6$ proinflammatory units, $\mathrm{p}=0.03$; Figure $5 \mathrm{E}$ ). Furthermore, when separating GBMs regardless of IDH-mutation status into those with higher and lower pro-inflammatory scores according to median split, patients with higher GAMM pro-inflammatory status had longer overall survival ( $847.8 \pm 182.4$ days compared to $367.8 \pm 63.2$ days, respectively, $\mathrm{p}=0.03$; Figure $5 \mathrm{~F}$ ). Notably, all IDHmutant GBMs had a higher pro-inflammatory profile.

\section{The pro-inflammatory innate immune phenotype of IDH-MUT GBMs is driven by microglia}

To further understand the innate immune phenotype, we examined scRNA-seq databases. Since microglia and macrophages cannot be reliably distinguished at the protein level, we sought to determine their relative contribution to the GBM microenvironment by directly comparing the only available IDH-MUT GBM scRNAseq database with another containing IDH-WT GBMs. A total of 1,004 cells were available for analysis (569 from untreated IDH-WT GBMs, 270 from a treated IDH-MUT 
A

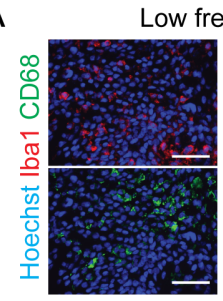

C

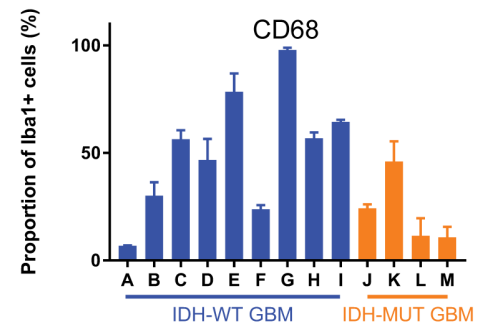

E

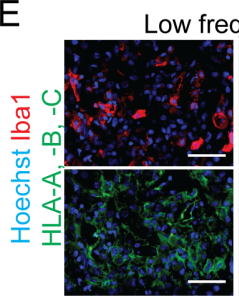

G

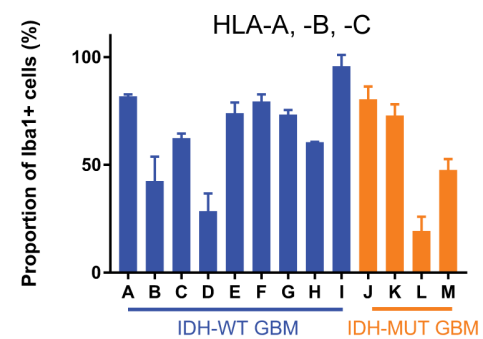

I

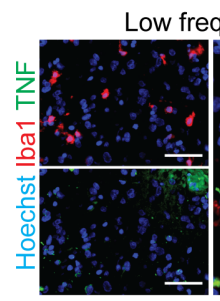

equency in Iba1+ cells

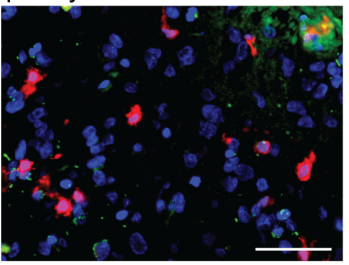

$\mathrm{K}$

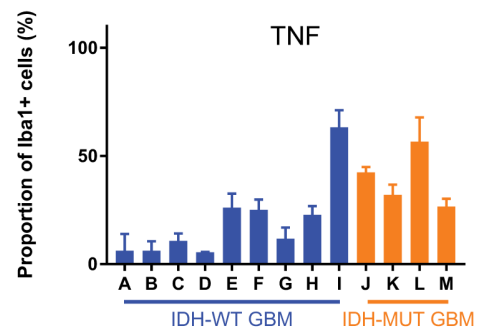

B

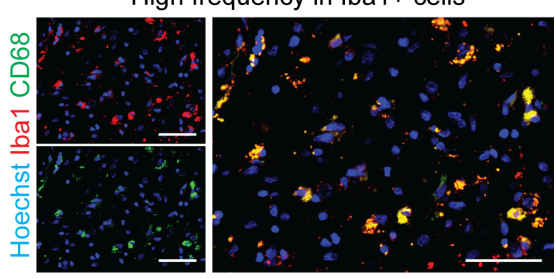

$\mathrm{D}$ ङ ${ }^{100} 7 \quad$ CD68

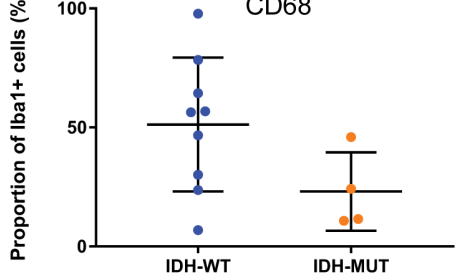

$\mathrm{F}$

F High frequency in Iba $1+$ cells

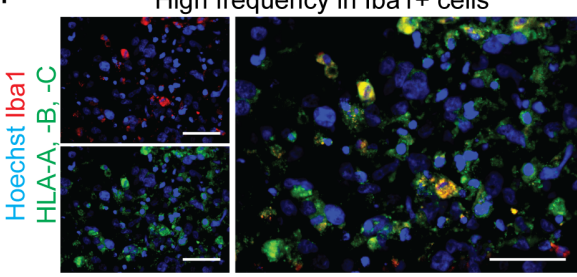

$\mathrm{H}$

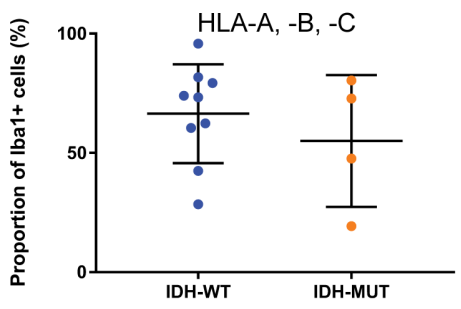

$J$

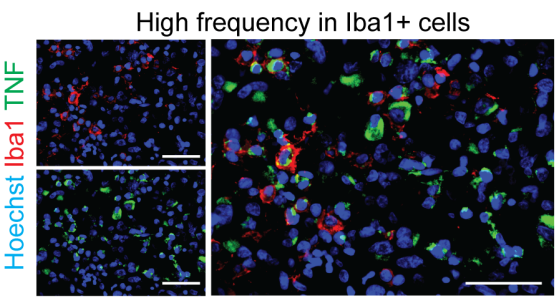

$\mathrm{L}$

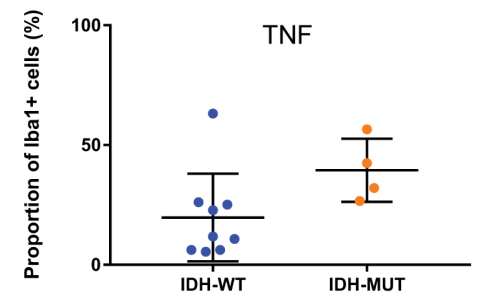

Figure 3: The expression of pro-inflammatory markers is highly variable in GAMMs. (A, B) Representative images of low (A) and high (B) frequency of the pro-inflammatory marker CD68 in Iba1+ microglia and macrophages of different GBMs. (C, D) Graphical representation of the results in (A and $B), p=0.09$. (E, F) Representative images of low $(E)$ and high (F) frequency of the proinflammatory marker HLA-A, -B, -C in microglia and macrophages of different GBMs. (G, H) Graphical representation of the results in $\mathrm{E}$ and $\mathrm{F}(\mathrm{p}=0.42)$. (I, J) Representative images of low (I) and high ( $\mathrm{J}$ ) frequency of the pro-inflammatory marker TNF in microglia and macrophages of different GBMs. $(\mathbf{K}, \mathbf{L})$ Graphical representation of the results in (I and J), p $=0.08$. Scale bars are $50 \mu \mathrm{m}$. 
A

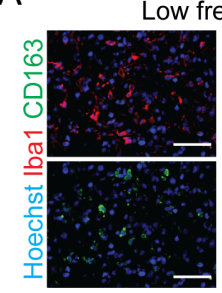

C

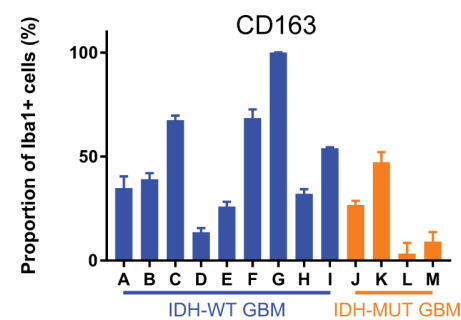

E

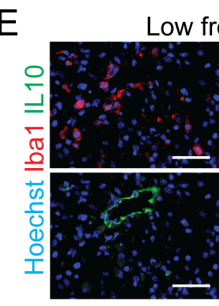

G

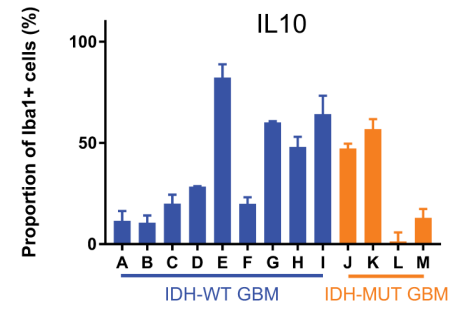

I

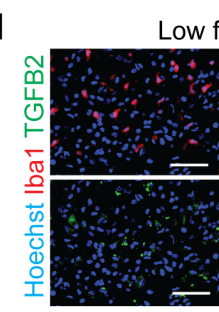

Low frequency in Iba1+ cells

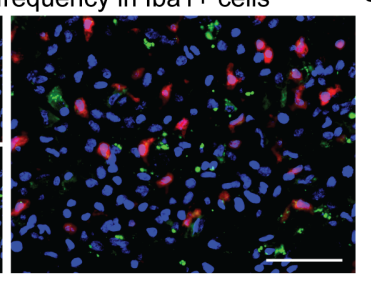

K

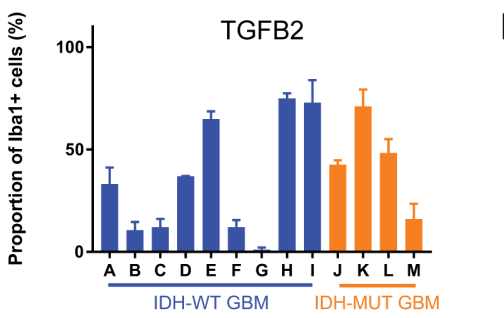

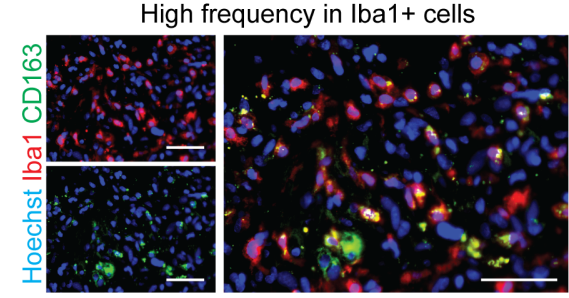

D

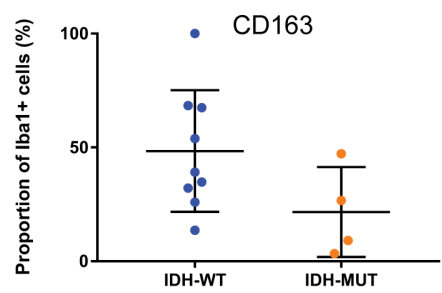

F

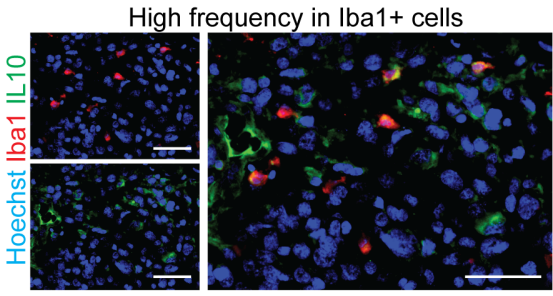

$\mathrm{H}$

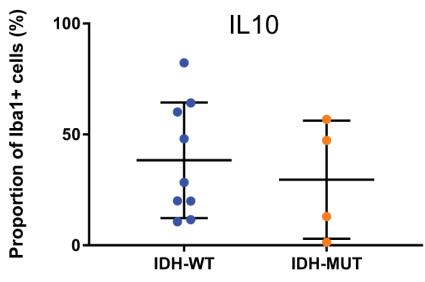

J
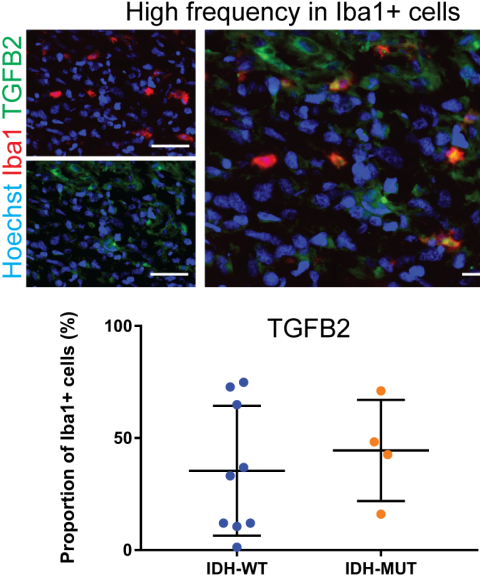

Figure 4: Anti-inflammatory markers are differentially expressed by GAMMs. (A, B) Representative images of low (A) and high (B) frequency of the anti-inflammatory marker CD163 in microglia and macrophages of different GBMs. (C, D) Graphical representation of the results in A and $B(p=0.10)$. $(\mathbf{E}, \mathbf{F})$ Representative images of low $(\mathrm{E})$ and high $(\mathrm{F})$ frequency of the anti-inflammatory marker IL10 in microglia and macrophages of different GBMs. (G, H) Graphical representation of the results in E and F (p=0.59). (I, J) Representative images of low (I) and high (J) frequency of the anti-inflammatory marker TGFB2 in microglia and macrophages of different GBMs. $(\mathbf{K}, \mathbf{L})$ Graphical representation of the results in I and $\mathbf{J}(\mathrm{p}=0.59)$. Scale bars are $50 \mu \mathrm{m}$. 
GBM [identified as MGH45 in GEO accession number GSE89567], and 165 from an untreated IDH-MUT GBM [identified as MGH57 in GEO GSE89567]). We then separated scRNA-seq libraries via clustering by gene expression using both $\mathrm{t}$-distributed stochastic neighbor embedding (tSNE; Figure 6A) and principal component analysis (PCA; Figure 6B and Supplementary Figure 2) techniques. Regardless of separation method, Clusters 1 and 7 segregated together, as well as Clusters 2 and 6 . Absolute contributions of cells from each database are shown in Figure 6C.
Gene enrichment analyses based on the microglia and macrophage curated gene list revealed that Clusters 1 ( 79 cells from the treated IDH-MUT GBM, 165 cells from the untreated IDH-MUT GBM), 4 (117 cells from the IDHWT GBMs), 5 (112 cells from the untreated IDH-MUT GBM), and 7 (68 cells from the treated IDH-MUT GBM) were highly enriched for microglia and/or macrophage genes (Figure 6D and Supplementary Table 4). Ingenuity pathway analysis (IPA) agreed with these designations by showing that the remaining clusters (Clusters 2, 3, and 6) had upregulation of canonical non-immune pathways
A
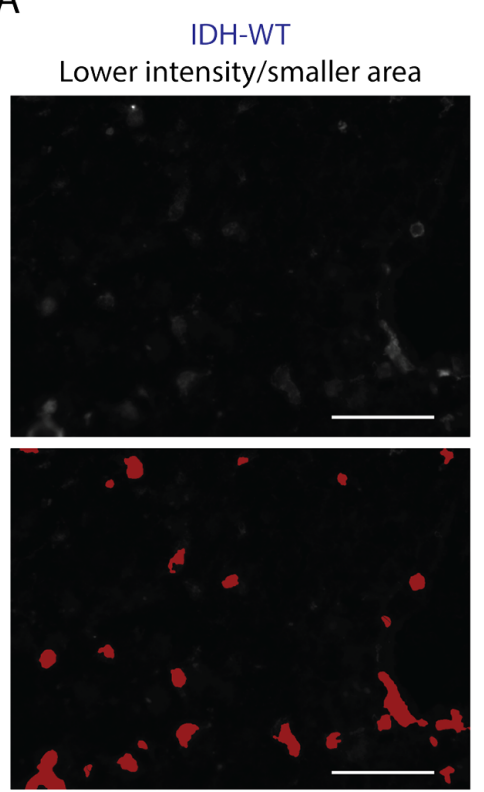

B

IDH-MUT
Higher intensity/ larger area
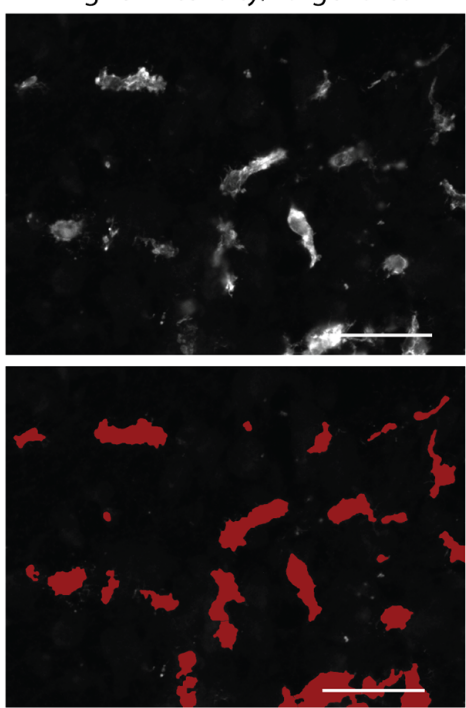

$\mathrm{E}$
C
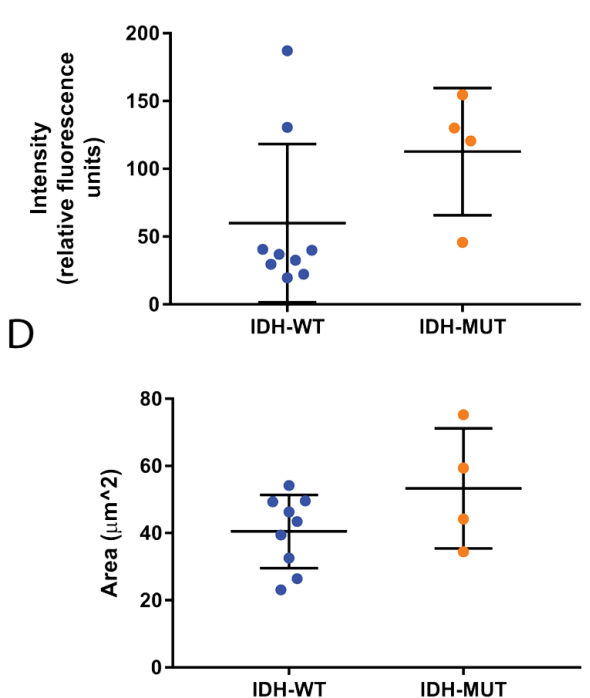

$\mathrm{F}$

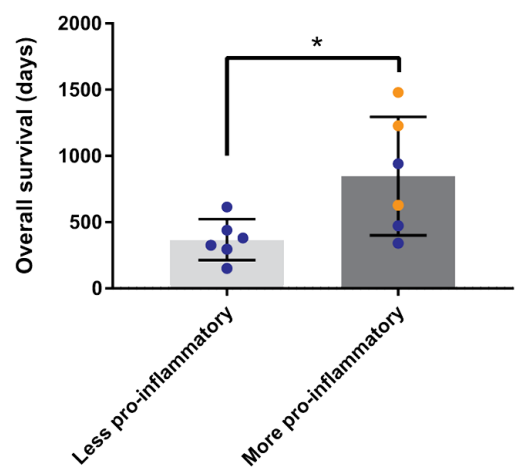

Figure 5: Microglia and macrophages are more pro-inflammatory in IDH-MUT compared to IDH-WT GBMs. (A, B) Representative images and corresponding overlays generated from the automated segmentation protocol of Iba1 intensity (A) and surface area (B) differences between IDH-WT and -MUT glioblastomas. Scale bars are $50 \mu \mathrm{m}$. (C, D) Graphical representation of the statistical comparison between A and $B(p=0.14$ and $p=0.13$, respectively). (E) Graphical representation of the activation profile revealing that IDH-MUT glioblastomaassociated microglia and macrophages are more pro-inflammatory than IDH-WT GAMMs $(\mathrm{p}=0.03)$. The activation profile was compiled by tabulating the overall inflammatory status, the Ibal intensity, and the surface area of cells. (F) Overall survival is significantly greater in GBMs with higher activation profiles as determined by median split without regard to IDH status $(\mathrm{p}=0.03)$. Nonetheless, values obtained from IDH-MUT glioblastomas (orange) fell into the higher pro-inflammatory and survival categories. One IDH-mutant GBM patient was lost to follow up leading to exclusion from this analysis. 
involved in metastasis, neuronal processes, and neoplasia, respectively (Supplementary Table 5).

Cluster 1 had higher expression of microglia $\left(\mathrm{p}=1.21 \times 10^{-10}, 12.2 \%\right.$ of microglia gene list $)$ than macrophage genes $\left(\mathrm{p}=1.68 \times 10^{-6}, 7.8 \%\right.$ of macrophage gene list, ) that were mostly pro-inflammatory $(\mathrm{p}=1.68 \times$ $10^{-6}, 5.8 \%$ of pro-inflammatory gene list; Supplementary Table 4). IPA revealed the most upregulated pathway was eukaryotic initiation factor 2 (EIF2) signaling $(\mathrm{p}=5.01 \times$ 10-14; Supplementary Table 5), a molecule shown to regulate pro-inflammatory cytokine expression [37]. This was similarly the most upregulated pathway in Cluster 7 $\left(\mathrm{p}=1 \times 10^{-8}\right.$; Supplementary Table 5), which was enriched with pro-inflammatory $\left(\mathrm{p}=1.01 \times 10^{-5}, 5.8 \%\right.$ of gene list $)$ microglia genes $\left(\mathrm{p}=2.18 \times 10^{-12}, 13.9 \%\right.$ of gene list $)$.

Cluster 4 was very significantly enriched with antiinflammatory $\left(\mathrm{p}=1.97 \times 10^{-89}, 6.4 \%\right.$ of anti-inflammatory gene list; Supplementary Table 4) macrophages $\left(p=3.18 \times 10^{-60}, 25.1 \%\right.$ of gene list $)$. Several pathways supporting this designation were strongly represented including those involved in communication between innate and adaptive immune cells $\left(\mathrm{p}=1 \times 10^{-12}\right)$, the antigen presentation pathway $\left(\mathrm{p}=2.14 \times{ }^{10-9}\right)$, and the most highly upregulated pathway dendritic cell maturation $(\mathrm{p}=1.58 \times$ $10^{-17}$; Supplementary Table 5). This pathway was linked to expression of genes such as $H L A-D R B 1, H L A-D R A$, and $F C G R 1 B$, molecules all known to be highly expressed in macrophages [38, 39].

Lastly, Cluster 5 contained microglia $(\mathrm{p}=9.98 \times$ $10^{-50}, 33.9 \%$ of gene list) and macrophages ( $\mathrm{p}=1.3 \times 10^{-}$ ${ }^{84}, 39.1 \%$ of gene list) that were mostly anti-inflammatory $\left(\mathrm{p}=4.64 \times 10^{-95}, 9.9 \%\right.$ of gene list), although there was a smaller pro-inflammatory representation $(\mathrm{p}=1.92 \times$ $10^{-47}, 9.9 \%$ of gene list; Supplementary Table 4). Again, the dendritic cell maturation pathway was highly upregulated $\left(\mathrm{p}=1.58 \times 10^{-17}\right)$ as well as the NFAT pathway which is known to be important in microglial and macrophage toll-like receptor signaling (Supplementary Table 5)[40, 41]. Please refer to Figure 6E for summarized inflammatory data.
A

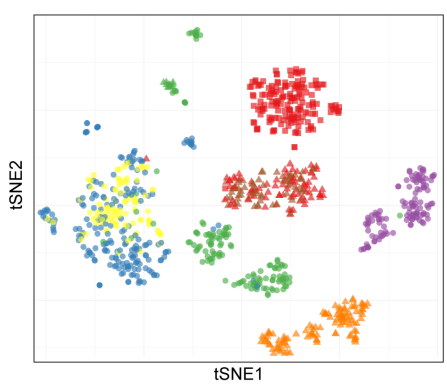

B

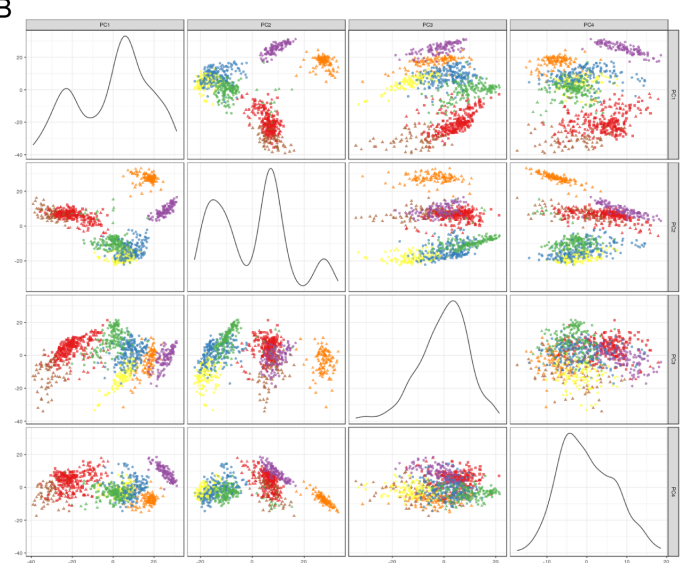

C

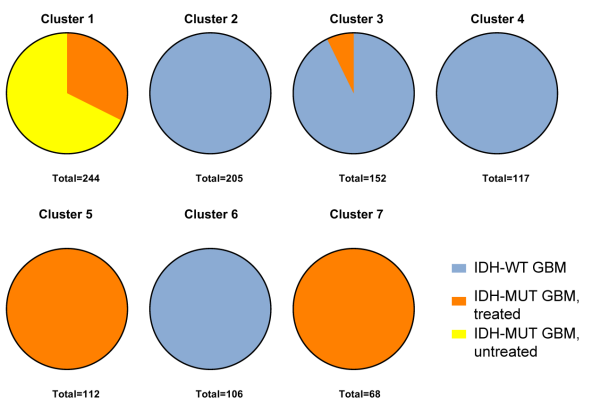

$\mathrm{D}$

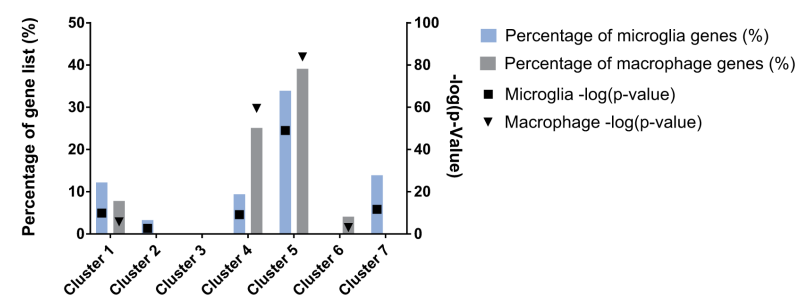

$\mathrm{E}$

\begin{tabular}{|c|c|c|c|c|}
\hline Cluster & $\begin{array}{l}\text { Anti-inflammatory } \\
\text { FDR-adjusted p-Value }\end{array}$ & $\begin{array}{c}\text { Anti-inflammatory } \\
\text { genes (\%) }\end{array}$ & $\begin{array}{l}\text { Pro-inflammatory FDF } \\
\text { adjusted p-value }\end{array}$ & $\begin{array}{l}\text { - Pro-inflammatory } \\
\text { genes (\%) }\end{array}$ \\
\hline 1 & $6.59 \mathrm{E}-04$ & 2.9 & $1.68 \mathrm{E}-06$ & 5.8 \\
\hline 4 & $1.97 \mathrm{E}-89$ & 6.4 & $1.52 \mathrm{E}-09$ & 5.4 \\
\hline 5 & $4.64 \mathrm{E}-95$ & 9.9 & $1.92 \mathrm{E}-47$ & 17.9 \\
\hline 7 & - & 0 & $1.01 \mathrm{E}-05$ & 5.8 \\
\hline
\end{tabular}

Figure 6: Comparison of scRNA-seq databases identifies microglia as the predominant source of pro-inflammatory milieu in IDH-MUT GBMs compared to their IDH-WT counterparts. (A) tSNE plot showing individual cell clusters generated according to gene expression values. (B) Principal component plots demonstrating clustering based on gene expression values. (C) The origin of cells according to data source is shown. Notice in both A and B Clusters 1 and 7 (innate immune cells) and Clusters 2 and 6 (presumed neoplastic cells) congregate together. (D) The corrected FDR p-value and percentage of curated microglia and macrophage gene list is graphically represented for each cluster. Highly significant representation of GAMMs is found in Clusters 1, 4, 5, and 7. (E) Further analysis of innate immune clusters reveals the anti- or pro-inflammatory expression of each cluster. The most pro-inflammatory GAMMs are found in the untreated IDH-MUT GBM. The treated IDH-MUT has a complement of both pro- and anti-inflammatory GAMMs. The IDH-WT GBMs have a predominance of anti-inflammatory macrophages while the IDH-MUT GBMs have a preponderance of proinflammatory microglia. 
Due to rigorous normalization processes that allowed data from one scRNA-seq database to be directly compared with another (for example, analysis of only cells in G1 phase, cells with at least 3,000 genes, etc.) [42], some data was excluded from analyses. Thus, only 165 data points from the untreated IDH-MUT GBM were included and we cannot confidently make statements about the quantity of microglia and macrophages since we do not know the denominator.

Overall, strongly anti-inflammatory macrophages were only found in IDH-WT and treated IDH-MUT GBMs. The untreated IDH-MUT GBM was represented mostly by pro-inflammatory microglia, which were also found in the treated IDH-MUT GBM.

\section{FCER1G, TYROBP, C1QA, C1QB, and CD74 were highly upregulated genes common to anti- inflammatory microglia and macrophages in Clusters 4 and 5}

Anti-inflammatory microglia and macrophages in Clusters 4 and 5 shared 5 out of 10 of the most upregulated genes in each cluster (Supplementary Table 4). Interestingly, all five of these genes, $C D 74$, $C 1 Q B, F C E R 1 G, T Y R O B P$, and CIQA are part of a protein-protein interaction network comprised of 20 proteins in total that are found only in head and neck squamous cell carcinomas but not in normal head and neck specimens [43]. Furthermore, FCERIG and TYROBP have been identified as two of three hub genes in a protein-protein interaction network positively correlated with the progression of clear cell renal cell carcinoma [44]. FCERIG and TYROBP encode for ITAM-containing adaptor proteins which may play major roles in CSF1R signaling [45], a receptor crucial to microglia and macrophage homeostasis and differentiation [46]. Similarly, C1QA and $C 1 Q B$ are involved in regulating IFN $\gamma$ signaling [47], a pathway crucial to pro-inflammatory cytokine secretion in microglia and macrophages. Lastly, $C D 74$, the migration inhibitory factor receptor that is expressed on GAMMs, has been described as a means by which IFN $\gamma$ signaling is disrupted, thus resulting in the promotion of an anti-inflammatory environment [48]. Unlike the anti-inflammatory genes, different pro-inflammatory genes were expressed by microglia in Clusters 1, 5, and 7. Altogether this suggests that there are targetable anti-inflammatory genes commonly upregulated by GAMMs that has relevance to other cancer types.

\section{DISCUSSION}

This is the first study to provide a head-to-head comparison between microglia and macrophages in human untreated IDH-MUT and -WT GBMs. In this report, we found that the innate immune microenvironment in these two categories of GBM was distinct. First, we showed that microglia and macrophage content was strikingly lower in IDH-MUT GBMs than their wild type counterparts. This is in line with a study in human IDH-mutated lower grade glioma and a syngeneic mouse model for IDH-MUT glioma which showed that downregulation of leukocyte chemotaxis contributed to less microglia and macrophage numbers in IDH-MUT tumors [49]. The lower amount of microglia and macrophages in IDH-MUT GBMs may either be a cause or consequence of GBM behavior. It would be important in the future to determine whether GAMM accumulation and associated activities promote malignancy, or whether their presence is a symptom of cancer-driven immunomodulation. The former would suggest immunotherapies targeting GAMM elimination or repulsion need to be developed, while the latter supports efforts to combat GBM-led immunomodulation for instance by re-stimulating GAMMs. Furthermore, decreased GAMM content in IDH-MUTs suggests immunotherapies aimed at activating innate immune cells would be more appropriately applied to IDH-WT GBMs.

In addition to differences in GAMM content, IDHMUT GAMMs also displayed a disparate activation profile from IDH-WT GAMMs. Most of the literature suggests that GBM creates a potently immunosuppressive microenvironment that influences microglia and macrophages to adopt and perpetuate similarly antiinflammatory profiles [12]. However, we show that the frequency of pro- and anti-inflammatory GAMMs varies drastically from GBM to GBM, especially in IDH-WTs, suggesting that levels of immunosuppression are also variable between tumors. The heterogeneity of our results makes the case for first profiling the innate immune milieu of a patient's GBM prior to immunotherapy selection. For instance, administering immunostimulants to GAMMs which are already pro-inflammatory would likely result in no benefit. The informed selection of immunotherapies during clinical trials is particularly important given the expense of getting new drugs to market and dilution of treatment effects by giving candidate therapies to nonresponders.

By considering multiple indicators of activation together, we find that IDH-MUT GAMMs possess more pro-inflammatory characteristics than IDH-WT GAMMs. Our scRNA-seq data mining of results from independent laboratories verifies this finding and suggests that the more pro-inflammatory GAMM in IDH-MUT results from microglia; conversely, the anti-inflammatory phenotype of GAMMs in IDH-WT GBMs appears to be driven by macrophages. Interestingly while different pro-inflammatory microglia clusters upregulated different pro-inflammatory genes, the anti-inflammatory genes that 
were the most highly upregulated were shared between clusters dominated by macrophages. Thus, these genes, FCER IG, TYROBP, C1QA, C1QB, and CD74 represent actionable targets for future therapy development. Interestingly, several of them are involved in the CSF1R and IFN $\gamma$ signaling pathways which play major roles in microglia and macrophage biology and activation. Overall, it appears that researching immunostimulants to promote pro-inflammatory phenotypes in macrophages specifically is a promising endeavor. The fact that these cells start in the periphery as monocytes also suggests that immunostimulants could be administered systemically and may not necessarily need to penetrate the blood brain barrier.

A limitation of this study is the relatively small sample size. However, the untreated IDH-MUT GBM tissue is rare so this limitation is difficult to overcome. Nonetheless, our quantitation methods are automated and absolute instead of semi-quantitative or manual. Lastly, while techniques such as high-dimensional single-cell analyses using mass cytometry are now available and allow the use of more markers than our comparatively simple immunofluorescence techniques, the processing required to run a sample through the mass cytometer including enzymatic, chemical, and mechanical dissociation into single-cell suspensions intrinsically alters the phenotype of microglia and macrophages prior to data capture [50], which would obviate our goal of determining the naïve GAMM activation state.

In conclusion, the phenotype of GAMM differs between IDH-WT and -MUT GBMs. Our results show this novel distinction at the protein level in rare untreated human IDH-MUT GBMs and at the single-cell RNA level through public databases. Not only are substantially less microglia and macrophages found in mutants, but they are also more pro-inflammatory, a generally soughtafter state in GBM treatment because of its association with immunostimulatory and tumor-suppressing properties [51, 52]. Increased microglia and macrophage pro-inflammatory activation may help explain why GBM patients with IDH mutations fare better than those without. Indeed, a high pro-inflammatory score is associated with a better overall survival regardless of IDH mutation status. Lastly, another surprising conclusion is that the heterogeneity in GAMM number as well as inflammatory phenotype is diverse in IDHWT GBM where microglia and macrophage content can range from approximately 0 to $70 \%$. This makes a strong argument for precision medicine in GBM, particularly with regards to immunotherapy development and selection. This incredible GAMM variation highlights the importance of future research elucidating what hosttumor interactions lead to this marked diversity, and what mechanisms underlying the difference in MM biology between IDH-MUTs and -WTs can be exploited for therapeutic gain.

\section{MATERIALS AND METHODS}

\section{Collection of glioblastoma samples}

GBM tissue was obtained only from the gadoliniumenhancing region on pre-operative MRIs to preclude areas of gross necrosis (Figure 1) using Medtronic StealthStation Surgical Navigation. This was conducted in 13 patients with previously untreated GBM (9 IDH-WT and 4 IDH-MUT). Patient demographics are detailed in Supplementary Table 1. Surgical excision was performed in all cases by the same neurosurgeon (JJK). Diagnosis of GBM and determination of IDH status was made through histopathological review by board-certified neuropathologists. Tumor specimens and clinical data were obtained as per protocol approved by the local institutional review board and ethics committee and conducted in accordance with national regulations. All patients provided written informed consent.

\section{Flow cytometry}

GBM specimens fresh from the operating room were mechanically and enzymatically digested for one hour using collagenase (4 mg), DNAse (10 mg), and kynurenic acid (4 mg). The resultant single-cell suspensions were passed through a $40 \mu \mathrm{m}$ strainer and stained with Fixable Viability Stain 510 (1:1000; BD Horizon, \#564406), APC-Cy7-CD11b (1:250; BD Pharmingen, \#557657) and PE-Cy5-CD45 antibodies (1:250; BD Pharmingen, \#555484) or isotype controls

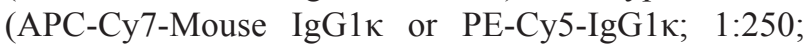
BD Pharmingen, \#557873 and \#555750, respectively). Samples were analyzed using either the BD FACSAria Fusion Cell Sorter or the Sony SH800. Data was processed using Flowjo software (Treestar). Debris, doublets, and dead cells were excluded using forward and side scatter parameters, and live cell gates. IDHMUT GBMs were acquired after flow cytometry protocol development, thus microglia and macrophage quantitation of these tumors was only with immunofluorescence.

\section{Tissue fixation and immunofluorescence}

GBM tissue from the operating room was immediately fixed in $4 \%$ paraformaldehyde for $15 \mathrm{~min}$, embedded in optimal cutting temperature compound, flash frozen and stored at $-80^{\circ} \mathrm{C}$ until use. Cryostat sections were cut at $10 \mu \mathrm{m}$ thickness and slide-mounted. Slides were permeabilized with $0.25 \%$ Triton X-100, blocked with $3 \%$ bovine serum albumin, and incubated with primary antibodies (Supplementary Table 2) at $4{ }^{\circ} \mathrm{C}$ overnight. The next day, slides were incubated with secondary antibodies (Supplementary Table 2) and counterstained with Hoechst at room temperature in the dark. 


\section{Imaging and analysis}

Immunofluorescent images were obtained with the ImageXpress Micro XLS widefield high-content analysis system and analyzed with MetaXpress 5 software using an in-house developed previously published protocol [21]. Briefly, a rectangular maximal region of interest on slidemounted sections was selected within tissue borders for imaging (Figure 1). Individual sections of $10 \mu \mathrm{m}$ thickness ranged from approximately $3000-8000 \mu \mathrm{m}$ in diameter (relatively large sections compared to those available in the literature). Each region of interest was divided into equally-sized rectangular sites of $225 \times 168 \mu \mathrm{m}$. After image acquisition, sites that included tissue folds, rents, regions of poor focus, and large blood vessels defined as > $25 \%$ of the site area were manually excluded (this typically represented $<10-15 \%$ of all sites). A no primary antibody control customized to individual GBMs was used to define the threshold above which staining was positive to account for highly variable levels of autofluorescence between GBM specimens. The threshold was set to allow no more than 5\% false-positive staining for each fluorophore used. Automated segmentation which allows for single-cell quantitation was performed using the MetaXpress Multiwavelength Cell Scoring Module according to user-defined parameters which included setting the minimum and maximum nuclear and cell diameter as well as the minimum area stained. Microglia and macrophage content was calculated with the formula:

number of Iba1+ cells/total nuclei $\times 100 \%$.

The microglia and macrophage inflammatory phenotype was calculated with the formula:

number of inflammatory marker and Iba1 doublepositive cells/number of Iba $1+$ cells $\times 100 \%$.

Ibal intensity and surface area were also quantitated in an automated fashion with MetaXpress using the same user-defined parameters. Image acquisition and analysis was performed for three consecutive sections of each GBM.

\section{Pro-inflammatory profile calculation}

The overall inflammatory profile (derived from subtracting the frequency of GAMM anti-inflammatory markers from pro-inflammatory markers), Iba1 intensity, and surface area of microglia and macrophages were chosen a priori as indicators of pro-inflammatory activation. To combine these parameters, each dataset was scaled by setting the minimum measured value to " 0 " and the maximum value to " 100 ", then summated. Patients that were still alive were given an overall survival as of May 23, 2018. One IDH-mutant patient was lost to follow up and this data was not included in the analysis.

\section{Statistical analysis}

All statistical analyses were performed with Microsoft Excel and Graphpad Prism. Results are shown as mean values \pm standard deviations. Statistical significance was calculated by a two-tailed Student's t-test. Correlation analysis was performed with Pearson's Correlation Formula. Statistical significance was defined at $\mathrm{p}<0.05$.

\section{Analysis of scRNA-seq databases}

Treated and untreated IDH-MUT GBM [10] singlecell gene expression levels were downloaded from the NCBI GEO repository under accession GSE89567. Single-cell gene expression levels for untreated IDH-WT GBMs [4] were generated from the NCBI SRA repository under accession SRP079058 by truncating the raw reads to 38bp pair-end data, then applying the same mapping, normalization and filtering procedures as described in Venteicher et al., 2017 [10]. All cells were assessed in the $\mathrm{R}$ statistical programming language for cell cycle stage using cyclone [42], and only G1 phase cells were used in subsequent analysis. Each subject's G1-phase cells were subjected to its own principal component analysis denoising procedure based on variance trends [53]. Denoised data from all three subjects were combined into a master dataset, and clustered based on Ward D2 criterion [54] for genes' log read counts. Dynamic Tree Cutting in R[55] was used with default parameters to generate the final cluster assignments. The "findMarkers" method of Scialdone et al. (2015)[42], was used to identify significantly differentially expressed genes $(\mathrm{FDR}<1 \times 10-20)$ in each cluster. Genes from each cluster that were upregulated at least 2-fold were submitted to Ingenuity Pathway Analysis (IPA; Qiagen, Redwood City, CA, USA) for canonical pathway and bespoke gene list enrichment analysis. FDR p-value correction was applied to all IPA results.

\section{Generation of gene lists and gene enrichment analysis}

Lists of genes that were differentially expressed between microglia and macrophages, and pro-versus antiinflammatory genes, were collected from all available human GBM single-cell RNA sequencing studies as of June 18, 2018 (Supplementary Table 3). Four manuscripts were included using these search criteria $[4,9,11]$. Duplicates were removed. If not already present, genes representing the six inflammatory markers investigated in this manuscript were added (CD68, HLA-A, -B, -C, TNF, CD163, IL10, $T G F B 2$ ). Differentially expressed genes generated in the aforementioned scRNA-seq were then cross-referenced with the curated gene lists and compared amongst clusters.

\section{Abbreviations}

GBM: glioblastoma; IDH: isocitrate dehydrogenase; WT: wildtype; MUT: mutant; scRNA-seq: single-cell RNA-sequencing; GAMM: glioblastoma-associated microglia and macrophages 


\section{Author contributions}

Conception of work: CCP, VWY, JJPK; experimental design: CCP, PMKG; data acquisition and analysis: CCP, PMKG, KL, RY, SS, RM, STA, MLH; manuscript writing and editing: CCP, VWY, JJPK; study supervision: VWY, JJPK.

\section{ACKNOWLEDGMENTS}

The authors wish to acknowledge the Hotchkiss Brain Institute Advanced Microscopy Platform facility and the University of Calgary Flow Cytometry Core Facility for the use of their equipment.

\section{CONFLICTS OF INTEREST}

No potential conflicts of interest were disclosed.

\section{FUNDING}

This project was funded by operating grants from the Canadian Institutes of Health Research and the Alberta Innovates, Health Solutions/Alberta Cancer Foundation.

\section{REFERENCES}

1. Stupp R, Mason WP, van den Bent MJ, Weller M, Fisher B, Taphoorn MJ, Belanger K, Brandes AA, Marosi C, Bogdahn U, Curschmann J, Janzer RC, Ludwin SK, et al. Radiotherapy plus concomitant and adjuvant temozolomide for glioblastoma. N Engl J Med. 2005; 352: 987-96. https:// doi.org/10.1056/NEJMoa043330.

2. Eggermont AMM, Blank CU, Mandala M, Long GV, Atkinson V, Dalle S, Haydon A, Lichinitser M, Khattak A, Carlino MS, Sandhu S, Larkin J, Puig S, et al. Adjuvant Pembrolizumab versus Placebo in Resected Stage III Melanoma. N Engl J Med. 2018; 378: 1789-801. https:// doi.org/10.1056/NEJMoa1802357.

3. Gandhi L, Rodriguez-Abreu D, Gadgeel S, Esteban E, Felip E, De Angelis F, Domine M, Clingan P, Hochmair MJ, Powell SF, Cheng SY, Bischoff HG, Peled N, et al. Pembrolizumab plus Chemotherapy in Metastatic NonSmall-Cell Lung Cancer. N Engl J Med. 2018; 378: 207892. https://doi.org/10.1056/NEJMoa1801005.

4. Darmanis S, Sloan SA, Croote D, Mignardi M, Chernikova S, Samghababi P, Zhang Y, Neff N, Kowarsky M, Caneda C, Li G, Chang SD, Connolly ID, et al. Single-Cell RNA-Seq Analysis of Infiltrating Neoplastic Cells at the Migrating Front of Human Glioblastoma. Cell Rep. 2017; 21: 1399410. https://doi.org/10.1016/j.celrep.2017.10.030.

5. Boddupalli CS, Bar N, Kadaveru K, Krauthammer M, Pornputtapong N, Mai Z, Ariyan S, Narayan D, Kluger H, Deng Y, Verma R, Das R, Bacchiocchi A, et al. Interlesional diversity of $\mathrm{T}$ cell receptors in melanoma with immune checkpoints enriched in tissue-resident memory $\mathrm{T}$ cells. JCI Insight. 2016; 1: e88955. https://doi.org/10.1172/jci. insight.88955.

6. Wakabayashi O, Yamazaki K, Oizumi S, Hommura F, Kinoshita I, Ogura S, Dosaka-Akita H, Nishimura M. $\mathrm{CD} 4+\mathrm{T}$ cells in cancer stroma, not $\mathrm{CD} 8+\mathrm{T}$ cells in cancer cell nests, are associated with favorable prognosis in human non-small cell lung cancers. Cancer Sci. 2003; 94: 1003-9.

7. Shinonaga M, Chang CC, Suzuki N, Sato M, Kuwabara T. Immunohistological evaluation of macrophage infiltrates in brain tumors. Correlation with peritumoral edema. Journal of Neurosurgery. 1988; 68: 259-65.

8. Morimura T, Neuchrist C, Kitz K, Budka H, Scheiner O, Kraft D, Lassmann H. Monocyte subpopulations in human gliomas: expression of $\mathrm{Fc}$ and complement receptors and correlation with tumor proliferation. Acta Neuropathologica. 1990; 80: 287-94.

9. Wang Q, Hu B, Hu X, Kim H, Squatrito M, Scarpace L, deCarvalho AC, Lyu S, Li P, Li Y, Barthel F, Cho HJ, Lin $\mathrm{YH}$, et al. Tumor Evolution of Glioma-Intrinsic Gene Expression Subtypes Associates with Immunological Changes in the Microenvironment. Cancer Cell. 2017; 32: 42-56 e6. https://doi.org/10.1016/j.ccell.2017.06.003.

10. Venteicher AS, Tirosh I, Hebert C, Yizhak K, Neftel C, Filbin MG, Hovestadt V, Escalante LE, Shaw ML, Rodman C, Gillespie SM, Dionne D, Luo CC, et al. Decoupling genetics, lineages, and microenvironment in IDH-mutant gliomas by single-cell RNA-seq. Science. 2017; 355: 31. https://doi.org/10.1126/science.aai8478.

11. Muller S, Kohanbash G, Liu SJ, Alvarado B, Carrera D, Bhaduri A, Watchmaker PB, Yagnik G, Di Lullo E, Malatesta M, Amankulor NM, Kriegstein AR, Lim DA, et al. Single-cell profiling of human gliomas reveals macrophage ontogeny as a basis for regional differences in macrophage activation in the tumor microenvironment. Genome Biol. 2017; 18: 234. https://doi.org/10.1186/s13059-017-1362-4.

12. Poon CC, Sarkar S, Yong VW, Kelly JJP. Glioblastomaassociated microglia and macrophages: targets for therapies to improve prognosis. Brain. 2017; 140: 1548-60. https:// doi.org/10.1093/brain/aww355.

13. Louis DN, Perry A, Reifenberger G, von Deimling A, Figarella-Branger D, Cavenee WK, Ohgaki H, Wiestler OD, Kleihues P, Ellison DW. The 2016 World Health Organization Classification of Tumors of the Central Nervous System: a summary. Acta Neuropathol. 2016; 131: 803-20. https://doi.org/10.1007/s00401-016-1545-1.

14. Baumert BG, Hegi ME, van den Bent MJ, von Deimling A, Gorlia T, Hoang-Xuan K, Brandes AA, Kantor G, Taphoorn MJB, Hassel MB, Hartmann C, Ryan G, Capper $\mathrm{D}$, et al. Temozolomide chemotherapy versus radiotherapy in high-risk low-grade glioma (EORTC 22033-26033): a randomised, open-label, phase 3 intergroup study. Lancet Oncol. 2016; 17: 1521-32. https://doi.org/10.1016/ S1470-2045(16)30313-8. 
15. Turcan S, Makarov V, Taranda J, Wang Y, Fabius AWM, Wu W, Zheng Y, El-Amine N, Haddock S, Nanjangud G, LeKaye HC, Brennan C, Cross J, et al. Mutant-IDH1dependent chromatin state reprogramming, reversibility, and persistence. Nat Genet. 2018; 50: 62-72. https://doi. org/10.1038/s41588-017-0001-z.

16. Eckel-Passow JE, Lachance DH, Molinaro AM, Walsh KM, Decker PA, Sicotte H, Pekmezci M, Rice T, Kosel ML, Smirnov IV, Sarkar G, Caron AA, Kollmeyer TM, et al. Glioma Groups Based on 1p/19q, IDH, and TERT Promoter Mutations in Tumors. N Engl J Med. 2015; 372: 2499-508. https://doi.org/10.1056/NEJMoa1407279.

17. Yan H, Parsons DW, Jin G, McLendon R, Rasheed BA, Yuan W, Kos I, Batinic-Haberle I, Jones S, Riggins GJ, Friedman H, Friedman A, Reardon D, et al. IDH1 and IDH2 mutations in gliomas. N Engl J Med. 2009; 360: 765-73. https://doi.org/10.1056/NEJMoa0808710.

18. Hide T, Komohara Y, Miyasato Y, Nakamura H, Makino K, Takeya M, Kuratsu JI, Mukasa A, Yano S. Oligodendrocyte Progenitor Cells and Macrophages/Microglia Produce Glioma Stem Cell Niches at the Tumor Border. EBioMedicine. 2018; 30: 94-104. https://doi.org/10.1016/j. ebiom.2018.02.024.

19. Chen H, Chong ZZ, De Toledo SM, Azzam EI, Elkabes S, Souayah N. Delayed activation of human microglial cells by high dose ionizing radiation. Brain Res. 2016; 1646: 193-8. https://doi.org/10.1016/j.brainres.2016.06.002.

20. Li W, Graeber MB. The molecular profile of microglia under the influence of glioma. Neuro Oncol. 2012; 14: 95878. https://doi.org/10.1093/neuonc/nos116.

21. Poon CC, Ebacher V, Liu K, Yong VW, Kelly JJP. Automated Slide Scanning and Segmentation in Fluorescently-labeled Tissues Using a Widefield Highcontent Analysis System. J Vis Exp. 2018. https://doi. org/10.3791/57440.

22. Hendrickx DAE, van Eden CG, Schuurman KG, Hamann J, Huitinga I. Staining of HLA-DR, Iba1 and CD68 in human microglia reveals partially overlapping expression depending on cellular morphology and pathology. J Neuroimmunol. 2017; 309: 12-22. https://doi.org/10.1016/j. jneuroim.2017.04.007.

23. Razavi SM, Lee KE, Jin BE, Aujla PS, Gholamin S, Li G. Immune Evasion Strategies of Glioblastoma. Front Surg. 2016; 3: 11. https://doi.org/10.3389/fsurg.2016.00011.

24. Liddelow SA, Guttenplan KA, Clarke LE, Bennett FC, Bohlen CJ, Schirmer L, Bennett ML, Munch AE, Chung WS, Peterson TC, Wilton DK, Frouin A, Napier BA, et al. Neurotoxic reactive astrocytes are induced by activated microglia. Nature. 2017; 541: 481-7. https://doi. org/10.1038/nature21029.

25. Ries CH, Cannarile MA, Hoves S, Benz J, Wartha K, Runza V, Rey-Giraud F, Pradel LP, Feuerhake F, Klaman I, Jones T, Jucknischke U, Scheiblich S, et al. Targeting tumor-associated macrophages with anti-CSF-1R antibody reveals a strategy for cancer therapy. Cancer Cell. 2014; 25 : 846-59. https://doi.org/10.1016/j.ccr.2014.05.016.

26. Lisi L, Ciotti GM, Braun D, Kalinin S, Curro D, Dello Russo C, Coli A, Mangiola A, Anile C, Feinstein DL, Navarra P. Expression of iNOS, CD163 and ARG-1 taken as M1 and M2 markers of microglial polarization in human glioblastoma and the surrounding normal parenchyma. Neurosci Lett. 2017; 645: 106-12. https://doi.org/10.1016/j. neulet.2017.02.076.

27. Ip WKE, Hoshi N, Shouval DS, Snapper S, Medzhitov R. Anti-inflammatory effect of IL-10 mediated by metabolic reprogramming of macrophages. Science. 2017; 356: 513-9. https://doi.org/10.1126/science.aal3535.

28. Bohlen CJ, Bennett FC, Tucker AF, Collins HY, Mulinyawe SB, Barres BA. Diverse Requirements for Microglial Survival, Specification, and Function Revealed by DefinedMedium Cultures. Neuron. 2017; 94: 759-73 e8. https://doi. org/10.1016/j.neuron.2017.04.043.

29. Christofides A, Kosmopoulos M, Piperi C. Pathophysiological mechanisms regulated by cytokines in gliomas. Cytokine. 2015; 71: 377-84. https://doi. org/10.1016/j.cyto.2014.09.008.

30. Han J, Alvarez-Breckenridge CA, Wang QE, Yu J. TGFbeta signaling and its targeting for glioma treatment. Am J Cancer Res. 2015; 5: 945-55.

31. Ito D, Tanaka K, Suzuki S, Dembo T, Fukuuchi Y. Enhanced expression of Iba1, ionized calcium-binding adapter molecule 1, after transient focal cerebral ischemia in rat brain. Stroke. 2001; 32: 1208-15.

32. Rawji KS, Kappen J, Tang W, Teo W, Plemel JR, Stys PK, Yong VW. Deficient Surveillance and Phagocytic Activity of Myeloid Cells Within Demyelinated Lesions in Aging Mice Visualized by Ex Vivo Live Multiphoton Imaging. J Neurosci. 2018; 38: 1973-88. https://doi.org/10.1523/ JNEUROSCI.2341-17.2018.

33. Dheen ST, Kaur C, Ling EA. Microglial activation and its implications in the brain diseases. Curr Med Chem. 2007; 14: 1189-97.

34. Kierdorf K, Prinz M. Factors regulating microglia activation. Front Cell Neurosci. 2013; 7: 44. https://doi. org/10.3389/fncel.2013.00044.

35. Glass CK, Natoli G. Molecular control of activation and priming in macrophages. Nat Immunol. 2016; 17: 26-33. https://doi.org/10.1038/ni.3306.

36. Mullard A. Microglia-targeted candidates push the Alzheimer drug envelope. Nat Rev Drug Discov. 2018; 17 : 303-5. https://doi.org/10.1038/nrd.2018.65.

37. Shrestha N, Bahnan W, Wiley DJ, Barber G, Fields KA, Schesser K. Eukaryotic initiation factor 2 (eIF2) signaling regulates proinflammatory cytokine expression and bacterial invasion. J Biol Chem. 2012; 287: 28738-44. https://doi.org/10.1074/jbc.M112.375915. 
38. Costabel U, Bross KJ, Andreesen R, Matthys H. HLA-DR antigens on human macrophages from bronchoalveolar lavage fluid. Thorax. 1986; 41: 261-5.

39. Rapino F, Robles EF, Richter-Larrea JA, Kallin EM, Martinez-Climent JA, Graf T. C/EBPalpha induces highly efficient macrophage transdifferentiation of B lymphoma and leukemia cell lines and impairs their tumorigenicity. Cell Rep. 2013; 3: 1153-63. https://doi.org/10.1016/j. celrep.2013.03.003.

40. Nagamoto-Combs K, Combs CK. Microglial phenotype is regulated by activity of the transcription factor, NFAT (nuclear factor of activated $\mathrm{T}$ cells). J Neurosci. 2010; 30: 9641-6. https://doi.org/10.1523/ JNEUROSCI.0828-10.2010.

41. Minematsu H, Shin MJ, Celil Aydemir AB, Kim KO, Nizami SA, Chung GJ, Lee FY. Nuclear presence of nuclear factor of activated T cells (NFAT) c3 and c4 is required for Toll-like receptor-activated innate inflammatory response of monocytes/macrophages. Cell Signal. 2011; 23: 1785-93. https://doi.org/10.1016/j.cellsig.2011.06.013.

42. Scialdone A, Natarajan KN, Saraiva LR, Proserpio V, Teichmann SA, Stegle O, Marioni JC, Buettner F. Computational assignment of cell-cycle stage from singlecell transcriptome data. Methods. 2015; 85: 54-61. https:// doi.org/10.1016/j.ymeth.2015.06.021.

43. Kim P, Cheng F, Zhao J, Zhao Z. ccmGDB: a database for cancer cell metabolism genes. Nucleic Acids Res. 2016; 44: D959-68. https://doi.org/10.1093/nar/gkv1128.

44. Chen L, Yuan L, Wang Y, Wang G, Zhu Y, Cao R, Qian G, Xie C, Liu X, Xiao Y, Wang X. Co-expression network analysis identified FCER1G in association with progression and prognosis in human clear cell renal cell carcinoma. Int J Biol Sci. 2017; 13: 1361-72. https://doi.org/10.7150/ ijbs.21657.

45. Forabosco P, Ramasamy A, Trabzuni D, Walker R, Smith C, Bras J, Levine AP, Hardy J, Pocock JM, Guerreiro R, Weale ME, Ryten M. Insights into TREM2 biology by network analysis of human brain gene expression data. Neurobiol Aging. 2013; 34: 2699-714. https://doi.org/10.1016/j. neurobiolaging.2013.05.001.

46. Pridans C, Raper A, Davis GM, Alves J, Sauter KA, Lefevre L, Regan T, Meek S, Sutherland L, Thomson AJ, Clohisey S, Bush SJ, Rojo R, et al. Pleiotropic Impacts of Macrophage and Microglial Deficiency on Development in Rats with Targeted Mutation of the Csf1r Locus. J Immunol. 2018; 201: 2683-99. https://doi.org/10.4049/ jimmunol.1701783.
47. Chen G, Tan CS, Teh BK, Lu J. Molecular mechanisms for synchronized transcription of three complement $\mathrm{C} 1 \mathrm{q}$ subunit genes in dendritic cells and macrophages. J Biol Chem. 2011; 286: 34941-50. https://doi.org/10.1074/jbc. M111.286427.

48. Ghoochani A, Schwarz MA, Yakubov E, Engelhorn T, Doerfler A, Buchfelder M, Bucala R, Savaskan NE, Eyupoglu IY. MIF-CD74 signaling impedes microglial M1 polarization and facilitates brain tumorigenesis. Oncogene. 2016; 35: 6246-61. https://doi.org/10.1038/onc.2016.160.

49. Amankulor NM, Kim Y, Arora S, Kargl J, Szulzewsky F, Hanke M, Margineantu DH, Rao A, Bolouri H, Delrow J, Hockenbery D, Houghton AM, Holland EC. Mutant IDH1 regulates the tumor-associated immune system in gliomas. Genes Dev. 2017; 31: 774-86. https://doi.org/10.1101/ gad.294991.116.

50. Haimon Z, Volaski A, Orthgiess J, Boura-Halfon S, Varol D, Shemer A, Yona S, Zuckerman B, David E, Chappell-Maor L, Bechmann I, Gericke M, Ulitsky I, et al. Re-evaluating microglia expression profiles using RiboTag and cell isolation strategies. Nat Immunol. 2018. https://doi. org/10.1038/s41590-018-0110-6.

51. Sarkar S, Doring A, Zemp FJ, Silva C, Lun X, Wang X, Kelly J, Hader W, Hamilton M, Mercier P, Dunn JF, Kinniburgh D, van Rooijen N, et al. Therapeutic activation of macrophages and microglia to suppress brain tumorinitiating cells. Nat Neurosci. 2014; 17: 46-55. https://doi. org/10.1038/nn.3597.

52. Wang Q, He Z, Huang M, Liu T, Wang Y, Xu H, Duan H, Ma P, Zhang L, Zamvil SS, Hidalgo J, Zhang Z, O'Rourke $\mathrm{DM}$, et al. Vascular niche IL-6 induces alternative macrophage activation in glioblastoma through HIF2alpha. Nat Commun. 2018; 9: 559. https://doi.org/10.1038/ s41467-018-03050-0.

53. Lun AT, McCarthy DJ, Marioni JC. A step-by-step workflow for low-level analysis of single-cell RNA-seq data with Bioconductor. F1000Res. 2016; 5: 2122. https:// doi.org/10.12688/f1000research.9501.2.

54. Murtagh F, Legendre P. Ward's Hierarchical Agglomerative Clustering Method: Which Algorithms Implement Ward's Criterion? Journal of Classification. 2014; 31: 274-95. https://doi.org/10.1007/s00357-014-9161-z.

55. Langfelder P, Zhang B, Horvath S. Defining clusters from a hierarchical cluster tree: the Dynamic Tree Cut package for R. Bioinformatics. 2008; 24: 719-20. https://doi. org/10.1093/bioinformatics/btm563. 